\title{
SPANNING SIMPLICIAL COMPLEXES OF $n$-CYCLIC GRAPHS WITH A COMMON VERTEX
}

\author{
Yan Pan, Rong Li and Guangjun Zhu \\ Received: 20 June 2014; Revised: 17 August 2014 \\ Communicated by Abdullah Harmancı
}

\begin{abstract}
In this paper, we characterize some algebraic and combinatorial properties of spanning simplicial complex $\Delta_{s}\left(G_{l_{1}}, l_{2}, \cdots, l_{n}\right)$ of the $n$-cyclic graphs $G_{l_{1}, l_{2}, \cdots, l_{n}}$ with a common vertex. We show that $\Delta_{s}\left(G_{l_{1}, l_{2}, \cdots, l_{n}}\right)$ is pure simplicial complex of dimension $\sum_{i=1}^{n} l_{i}-n-1$. We determine the Stanley-Reisner ideal $I_{\Delta_{s}}\left(G_{l_{1}, l_{2}, \ldots, l_{n}}\right)$ of $\Delta_{s}\left(G_{l_{1}, l_{2}, \cdots, l_{n}}\right)$ and its primary decomposition. Under the condition that the length of each cyclic graph is $t$, we also give a formula for $f$-vector of $\Delta_{s}\left(G_{l_{1}, l_{2}, \cdots, l_{n}}\right)$ and consequently

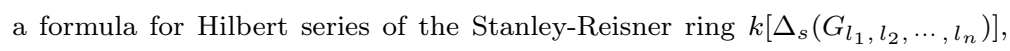
where $k$ is a field.
\end{abstract}

Mathematics Subject Classification (2010): 13P10, 13H10, 13F20, 13C14 Keywords: Spanning tree, simplicial complex, f-vector, h-vector, Hilbert series

\section{Introduction}

The note of spanning simplicial complex $\Delta_{s}(G)$ on edge set $E$ of a graph $G=$ $G(V, E)$ was introduced in [1], the set of its facets is exactly the edge set $s(G)$ of all possible spanning trees of $G$, i.e.

$$
\Delta_{s}(G)=\left\langle F_{i} \mid F_{i} \in s(G)\right\rangle
$$

Note that for a graph $\mathrm{G}$, the problem of finding $s(G)$ is not always easy to handle. Anwar, Raza and Kashif [1] proved some algebraic and combinatorial properties of spanning simplicial complex of the uni-cyclic graph $U_{n}$ (i.e., if the vertex set of $U_{n}$ is $V=\left\{x_{1}, \ldots, x_{n}\right\}$, then the edge set of $U_{n}$ is $E=\left\{x_{i} x_{i+1} \mid i=\right.$ $1, \ldots, n$, and $\left.x_{n+1}=x_{1}\right\}$ ). Zhu et al. [5] discussed some properties of the spanning simplicial complexes of the $n$-cyclic graphs with a common edge. In this paper,

The first author is supported by Jiangsu province university students' innovative plan ( 5731568313 ; 201310285083x). The third author is supported by the National Natural Science Foundation of China (11271275) and by Foundation of Jiangsu Overseas Research \& Training Program for University Prominent Young \& Middle-aged Teachers and Presidents. 
our goal is to characterize some algebraic and combinatorial properties of spanning simplicial complexes of some $n$-cyclic graphs $G_{l_{1}, l_{2}, \cdots, l_{n}}$ with a common vertex, which is obtained by joining $n$ disjoint cycles $G_{l_{1}}, \ldots, G_{l_{n}}$ of length $l_{1}, \ldots, l_{n}$ at a common vertex. For $n=2$ and $l_{1}=l_{2}=3$, the graph of $G_{3,3}$ is shown in Figure 1 of Section 2.

We give a brief overview of this paper. In Section 2, we recall some definitions and results from commutative algebra and algebraic combinatorics. In Section 3,

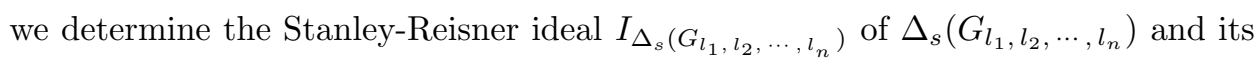
primary decomposition in Theorem 3.2. In Section 4, under the assumption that the length of every cycle is $t$, we give a formula for $f$-vector of $\Delta_{s}\left(G_{l_{1}, l_{2}, \cdots, l_{n}}\right)$ and consequently a formula for Hilbert series of the Stanley-Reisner $\operatorname{ring} k\left[\Delta_{s}\left(G_{l_{1}, l_{2}, \ldots, l_{n}}\right)\right]$.

\section{Preliminaries}

We firstly recall some definitions and basic facts about graph and simplicial complex in order to make this paper self-contained.

Definition 2.1. A spanning tree of a simple connected finite graph $G=G(V, E)$ is a subgraph of $G$, which is a tree and contains all vertices of $G$. We denote the collection of all edge sets of the spanning trees of $G$ by $s(G)$, i.e.

$s(G)=\left\{E\left(T_{i}\right) \subset E \mid T_{i}\right.$ is a spanning tree of $\left.G\right\}$ (See [3] for more details).

It is well known that for any simple connected finite graph, spanning trees always exist. One can find a spanning tree systematically by the cutting-down method, which says that a spanning tree is obtained by removing one edge from each cycle appearing in the graph. For example, for the following graph $G$, we obtain that

$$
\begin{aligned}
s(G)= & \left\{\left\{e_{2}, e_{3}, e_{5}, e_{6}\right\},\left\{e_{2}, e_{3}, e_{4}, e_{6}\right\},\left\{e_{2}, e_{3}, e_{4}, e_{5}\right\},\left\{e_{1}, e_{3}, e_{5}, e_{6}\right\},\left\{e_{1}, e_{3},\right.\right. \\
& \left.\left.e_{4}, e_{6}\right\},\left\{e_{1}, e_{3}, e_{4}, e_{5}\right\},\left\{e_{1}, e_{2}, e_{5}, e_{6}\right\},\left\{e_{1}, e_{2}, e_{4}, e_{6}\right\},\left\{e_{1}, e_{2}, e_{4}, e_{5}\right\}\right\}
\end{aligned}
$$

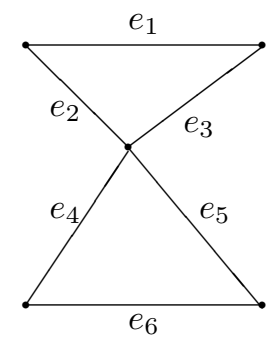

Figure 1. 2-cyclic graph with a common vertex

Definition 2.2. A simplicial complex $\Delta$ on a set of vertices $[n]=\{1,2, \ldots, n\}$ is a collection of subsets of $[n]$ such that 
(1) $\{i\} \in \Delta$ for each $i \in[n]$;

(2) if $F \in \Delta$ and $G \subseteq F$, then $G \in \Delta$.

An element of $\Delta$ is called a face of $\Delta$, and the dimension of a face $F$ of $\Delta$ is defined as $|F|-1$, where $|F|$ is the number of vertices of $F$ and denoted by $\operatorname{dim} F$. The faces of dimension 0 and 1 are called vertices and edges, respectively, and $\operatorname{dim} \emptyset=-1$.

The maximum faces of $\Delta$ under inclusion are called facets of $\Delta$. The dimension of the simplicial complex $\Delta$, which is denoted by $\operatorname{dim} \Delta$, is the maximal dimension of its facets, i.e.

$$
\operatorname{dim} \Delta=\max \{\operatorname{dim} F \mid F \text { is a facet of } \Delta\} .
$$

We denote the simplicial complex $\Delta$ with facets $\left\{F_{1}, \ldots, F_{q}\right\}$ by

$$
\Delta=\left\langle F_{1}, \ldots, F_{q}\right\rangle .
$$

Definition 2.3. A simplicial complex $\Delta$ is pure if all of its facets have the same dimension.

Definition 2.4. Given a simplicial complex $\Delta$ of dimension $d$, we define its $f$-vector to be the $(d+1)$-tuple $f=\left(f_{0}, f_{1}, \ldots, f_{d}\right)$, where $f_{i}$ is the number of $i$-dimensional faces of $\Delta$.

Definition 2.5. For a simple connected finite graph $G=G(V, E)$ with $s(G)=$ $\left\{E_{1}, \ldots, E_{s}\right\}$, we define a simplicial complex $\Delta_{s}(G)$ on $E$ such that facets of $\Delta_{s}(G)$ are precisely the elements of $s(G)$, called the spanning simplicial complex of $G(V, E)$. In other words,

$$
\Delta_{s}(G)=\left\langle E_{1}, \ldots, E_{s}\right\rangle .
$$

As the number of elements of both $E_{i}$ and $E_{j}$ are $|E|-m$, where $m$ denotes the number of cycles in $G$, we have that $E_{i} \nsubseteq E_{j}$ for $i \neq j$.

For example, the spanning simplicial complex of the graph $G$ with edge set $E=\left\{e_{1}, e_{2}, e_{3}, e_{4}, e_{5}, e_{6}\right\}$ in Figure 1 is given by

$$
\begin{aligned}
\Delta_{s}(G)= & \left\langle\left\{e_{2}, e_{3}, e_{5}, e_{6}\right\},\left\{e_{2}, e_{3}, e_{4}, e_{6}\right\},\left\{e_{2}, e_{3}, e_{4}, e_{5}\right\},\left\{e_{1}, e_{3}, e_{5}, e_{6}\right\},\left\{e_{1}, e_{3},\right.\right. \\
& \left.\left.e_{4}, e_{6}\right\},\left\{e_{1}, e_{3}, e_{4}, e_{5}\right\},\left\{e_{1}, e_{2}, e_{5}, e_{6}\right\},\left\{e_{1}, e_{2}, e_{4}, e_{6}\right\},\left\{e_{1}, e_{2}, e_{4}, e_{5}\right\}\right\rangle .
\end{aligned}
$$

Definition 2.6. An $n$-cyclic graph $G_{l_{1}, l_{2}, \ldots, l_{n}}$ with a common vertex is a graph which is obtained by joining $n$ disjoint cycles $G_{l_{1}}, G_{l_{2}}, \ldots, G_{l_{n}}$ at a common vertex, where $G_{l_{i}}$ denotes the cycle of length $l_{i}$ and $l_{i} \geq 3$ for each $i \in\{1,2, \ldots, n\}$.

Remark 2.7. It is easy to see $G_{l_{1}, l_{2}, \cdots, l_{n}}$ has $\sum_{i=1}^{n} l_{i}-n+1$ vertices and $\sum_{i=1}^{n} l_{i}$ edges. 


\section{Primary decomposition of $I_{\Delta_{s}\left(G_{l_{1}, l_{2}, \ldots, l_{n}}\right)}$}

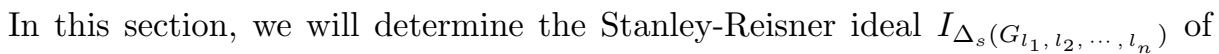
$\Delta_{s}\left(G_{l_{1}, l_{2}, \cdots, l_{n}}\right)$ and its primary decomposition.

We label the edge set of $G_{l_{1}, l_{2}, \ldots, l_{n}}$ such that $\left\{e_{i 1}, e_{i 2}, \ldots, e_{i l_{i}}\right\}$ is the edge set of the cycle $G_{l_{i}}$ for $1 \leq i \leq n$. First, we have the following proposition.

Proposition 3.1. $\Delta_{s}\left(G_{l_{1}, l_{2}, \cdots, l_{n}}\right)$ is a pure simplicial complex of dimension $\sum_{i=1}^{n} l_{i}-$ $n-1$.

Proof. Let $E=\left\{e_{11}, \ldots, e_{1 l_{1}}, e_{21}, \ldots, e_{2 l_{2}}, \ldots, e_{n 1}, \ldots, e_{n l_{n}}\right\}$ be the edge set of the $n$-cyclic graph $G_{l_{1}, l_{2}, \cdots, l_{n}}$. As $G_{l_{1}, l_{2}, \cdots, l_{n}}$ contains exactly n cycles of length $l_{1}, l_{2}, \ldots, l_{n}$, by the cutting-down method, its spanning trees are obtained by removing one edge from each cycle $G_{l_{i}}, 1 \leq i \leq n$. Hence, the subset $E\left(T_{i}\right) \subset E$ is in $s\left(G_{l_{1}, l_{2}, \ldots, l_{n}}\right)$ if and only if $E\left(T_{i}\right)=E \backslash\left\{e_{1 i_{1}}, \ldots, e_{n i_{n}}\right\}$ for some $i_{j} \in\left\{1, \ldots, l_{j}\right\}$, where $j$ runs from 1 to $n$, i.e.

$$
s\left(G_{l_{1}, l_{2}, \ldots, l_{n}}\right)=\left\{E \backslash\left\{e_{1 i_{1}}, \ldots, e_{n i_{n}}\right\} \mid i_{j} \in\left\{1, \ldots, l_{j}\right\} \text { and } j \in\{1, \ldots, n\}\right\} .
$$

It is easily seen that each spanning tree of $\Delta_{s}\left(G_{l_{1}, l_{2}, \cdots, l_{n}}\right)$ has $\sum_{i=1}^{n}\left(l_{i}-1\right)=\sum_{i=1}^{n} l_{i}-n$ edges, thus the result follows.

Let $E=\left\{e_{11}, \ldots, e_{1 l_{1}}, e_{21}, \ldots, e_{2 l_{2}}, \ldots, e_{n 1}, \ldots, e_{n l_{n}}\right\}$ be the edge set of the $n$ cyclic graph $G_{l_{1}, l_{2}, \cdots, l_{n}}$, and let $\Delta_{S}\left(G_{l_{1}, l_{2}, \cdots, l_{n}}\right)$ be the spanning simplicial complex of $G_{l_{1}, l_{2}, \ldots, l_{n}}$. We can assume that $S=k\left[x_{11}, \ldots, x_{1 l_{1}}, x_{21}, \ldots, x_{2 l_{2}}, \ldots, x_{n 1}, \ldots\right.$, $\left.x_{n l_{n}}\right]$ is a polynomial ring in $\sum_{i=1}^{n} l_{i}$ variables over a field $k, I_{\Delta_{s}\left(G_{l_{1}, l_{2}, \ldots, l_{n}}\right)}$ is the Stanley-Reisner ideal of $\Delta_{s}\left(G_{l_{1}, l_{2}, \ldots, l_{n}}\right)$, which is a squarefree monomial ideal. The standard graded algebra $k\left[\Delta_{s}\left(G_{l_{1}, l_{2}, \cdots, l_{n}}\right)\right]=S / I_{\Delta_{s}\left(G_{l_{1}, l_{2}, \cdots, l_{n}}\right)}$ is called the Stanley-Reisner ring of $\Delta_{s}\left(G_{l_{1}, l_{2}, \cdots, l_{n}}\right)$. We can give a primary decomposition of ideal $I_{\Delta_{s}\left(G_{l_{1}, l_{2}, \cdots, l_{n}}\right)}$, Hilbert series and $h$-vector of $k\left[\Delta_{s}\left(G_{l_{1}, l_{2}, \cdots, l_{n}}\right)\right]$. We refer readers to [2] and [4] for detailed information about the Stanley-Reisner ideal, primary decomposition and Hilbert series.

Now, we give a primary decomposition of the Stanley-Reisner ideal $I_{\Delta_{s}\left(G_{l_{1}, l_{2}}, \ldots, l_{n}\right)}$ of $\Delta_{s}\left(G_{l_{1}, l_{2}, \cdots, l_{n}}\right)$.

Theorem 3.2. Let $\Delta_{s}\left(G_{l_{1}, l_{2}, \cdots, l_{n}}\right)$ be the spanning simplicial complex of the $n$ cyclic graph $G_{l_{1}, l_{2}, \cdots, l_{n}}$. Then the Stanley-Reisner ideal $I_{\Delta_{s}\left(G_{l_{1}, l_{2}, \cdots, l_{n}}\right)}$ of 
$\Delta_{s}\left(G_{l_{1}, l_{2}, \cdots, l_{n}}\right)$ is given by

$$
\begin{aligned}
I_{\Delta_{s}\left(G_{l_{1}, l_{2}, \cdots, l_{n}}\right)}= & \bigcap_{\substack{i_{j} \in\left\{1,2, \ldots, l_{j}\right\} \\
j \in\{1,2, \ldots, n\}}}\left(x_{1 i_{1}}, x_{2 i_{2}}, \ldots, x_{n i_{n}}\right) \\
& =\left(x_{11} x_{12} \cdots x_{1 l_{1}}, x_{21} x_{22} \cdots x_{2 l_{2}}, \ldots, x_{n 1} x_{n 2} \cdots x_{n l_{n}}\right) .
\end{aligned}
$$

Proof. As each of facets of $\Delta_{s}\left(G_{l_{1}, l_{2}, \cdots, l_{n}}\right)$ is obtained by removing exactly one edge from each cycle $G_{l_{i}}, 1 \leq i \leq n$. From [4, Proposition 5.3.10], we get that

$$
\begin{aligned}
I_{\Delta_{s}\left(G_{l_{1}, l_{2}, \cdots, l_{n}}\right)}= & \bigcap_{\substack{i_{j} \in\left\{1,2, \ldots, l_{j}\right\} \\
j \in\{1,2, \ldots, n\}}}\left(x_{1 i_{1}}, x_{2 i_{2}}, \ldots, x_{n i_{n}}\right) \\
& =\left(x_{11} x_{12} \cdots x_{1 l_{1}}, x_{21} x_{22} \cdots x_{2 l_{2}}, \ldots, x_{n 1} x_{n 2} \cdots x_{n l_{n}}\right) .
\end{aligned}
$$

As corollaries, we obtain the following two results.

Corollary 3.3. Let $\Delta_{s}\left(G_{l_{1}, l_{2}, \cdots, l_{n}}\right)$ be the spanning simplicial complex of the $n$-cyclic graph $G_{l_{1}, l_{2}, \cdots, l_{n}}$. Then the Stanley-Reisner ring $S / I_{\Delta_{s}\left(G_{l_{1}, l_{2}}, \ldots, l_{n}\right)}$ is Gorenstein.

Proof. By the above theorem, we have that

$$
I_{\Delta_{s}\left(G_{l_{1}, l_{2}, \cdots, l_{n}}\right)}=\left(x_{11} x_{12} \cdots x_{1 l_{1}}, x_{21} x_{22} \cdots x_{2 l_{2}}, \ldots, x_{n 1} x_{n 2} \cdots x_{n l_{n}}\right) .
$$

It is clear that $x_{11} x_{12} \cdots x_{1 l_{1}}, x_{21} x_{22} \cdots x_{2 l_{2}}, \ldots, x_{n 1} x_{n 2} \cdots x_{n l_{n}}$ is a regular sequence in any order. As $S$ is Gorenstein, the Stanley-Reisner ring $S / I_{\Delta_{s}\left(G_{l_{1}, l_{2}, \cdots, l_{n}}\right)}$ is Gorenstein by [2, Proposition 3.1.19].

Corollary 3.4. Let $\Delta_{s}\left(G_{l_{1}, l_{2}, \cdots, l_{n}}\right)$ be the spanning simplicial complex of the $n$ cyclic graph $G_{l_{1}, l_{2}, \cdots, l_{n}}$. Then the Stanley-Reisner ideal $I_{\Delta_{s}\left(G_{l_{1}, l_{2}, \cdots, l_{n}}\right)}$ is unmixed of height $n$.

\section{The computation of $f$-vector of $\Delta_{s}\left(G_{\left.l_{1}, l_{2}, \cdots, l_{n}\right)}\right.$}

In this section, we will give a formula for $f$-vector of $\Delta_{s}\left(G_{l_{1}, l_{2}, \cdots, l_{n}}\right)$ and consequently a formula for Hilbert series of the Stanley-Reisner ring $k\left[\Delta_{s}\left(G_{l_{1}, l_{2}, \cdots, l_{n}}\right)\right]$ under the assumption that the length of every cycle $G_{l_{i}}$ is $t$ for $1 \leq i \leq n$. But before this we need the following proposition, its proof can be seen in Proposition 2.2 of [1].

Proposition 4.1. For a simplicial complex $\Delta$ on $[n]$ of dimension $d$, if $f_{t}=\left(\begin{array}{c}n \\ t+1\end{array}\right)$ for some $t \leq d$, then $f_{i}=\left(\begin{array}{c}n \\ i+1\end{array}\right)$ for all $0 \leq i<t$. 
Now, under the assumption that the length of every cycle $G_{l_{i}}$ is $t$ for $1 \leq i \leq n$, we give the formula to compute the $f$-vector of $\Delta_{s}\left(G_{l_{1}, l_{2}, \ldots, l_{n}}\right)$.

Theorem 4.2. Let $l_{i}=t$ for any $1 \leq i \leq n$. Then the $f$-vector of $\Delta_{s}\left(G_{l_{1}, l_{2}, \cdots, l_{n}}\right)$ is given by $f=\left(f_{0}, f_{1}, \ldots, f_{d}\right)$, where $d=n(t-1)-1$ and

$$
f_{j}=\sum_{i=0}^{k}(-1)^{i}\left(\begin{array}{c}
n \\
i
\end{array}\right)\left(\begin{array}{c}
n t-i t \\
j-i t+1
\end{array}\right),
$$

where $0 \leq j \leq d, k=\left[\frac{j+1}{t}\right]$ and symbol $[a], a \in Q$ denotes the maximum integer not exceeding $a$.

Proof. As $l_{i}=t$ for any $1 \leq i \leq n$, we can set $E=\left\{e_{11}, \ldots, e_{1 t}, e_{21}, \ldots, e_{2 t}, \ldots\right.$, $\left.e_{n 1}, \ldots, e_{n t}\right\}$ be the edge set of the $n$-cyclic graph $G_{l_{1}, l_{2}, \cdots, l_{n}}$. By the definition of $f$-vector of $\Delta_{s}\left(G_{l_{1}, l_{2}, \cdots, l_{n}}\right), f_{j}$ is the number of all those subsets of the edge set $E$ of the graph $G_{l_{1}, l_{2}, \ldots, l_{n}}$ with $j+1$ elements, that do not contain these cycles $\left\{e_{i_{1} 1}, \ldots, e_{i_{1} t} \mid 1 \leq i_{1} \leq n\right\},\left\{e_{i_{1} 1}, \ldots, e_{i_{1} t}, e_{i_{2} 1}, \ldots, e_{i_{2} t} \mid 1 \leq i_{1}<i_{2} \leq n\right\}, \ldots$, $\left\{e_{i_{1} 1}, \ldots, e_{i_{1} t}, \ldots, e_{i_{k} 1}, \ldots, e_{i_{k} t} \mid 1 \leq i_{1}<\cdots<i_{k} \leq n\right\}$.

By Remark 2.7, $G_{l_{1}, l_{2}}, \cdots, l_{n}$ has $n t$ edges, thus there are $\left(\begin{array}{c}n \\ k\end{array}\right)\left(\begin{array}{c}n t-k t \\ j-k t+1\end{array}\right)$ subsets of $E$ with $j+1$ elements, which contain the edge set $\left\{e_{i_{1} 1}, e_{i_{1} 2}, \ldots, e_{i_{1} t}, \ldots, e_{i_{k} 1}, \ldots, e_{i_{k} t} \mid\right.$ $\left.1 \leq i_{1}<\cdots<i_{k} \leq n\right\}$. Similarly, there are $\left(\begin{array}{c}n \\ k-1\end{array}\right)\left[\left(\begin{array}{c}n t-(k-1) t \\ j-(k-1) t+1\end{array}\right)-\left(\begin{array}{c}n-(k-1) \\ 1\end{array}\right)\left(\begin{array}{c}n t-k t \\ j-k t+1\end{array}\right)\right]$ $=\left(\begin{array}{c}n \\ k-1\end{array}\right)\left(\begin{array}{c}n t-k t+t \\ j-k t+t+1\end{array}\right)-\left(\begin{array}{c}n \\ k-1\end{array}\right)\left(\begin{array}{c}n-k+1 \\ 1\end{array}\right)\left(\begin{array}{c}n t-k t \\ j-k t+1\end{array}\right)=\sum_{i=0}^{1}(-1)^{i}\left(\begin{array}{c}n \\ k-1\end{array}\right)\left(\begin{array}{c}n-k+1 \\ i\end{array}\right)\left(\begin{array}{c}n t-(k-1+i) t \\ j-(k-1+i) t+1\end{array}\right)$ subsets of $E$ with $j+1$ elements, containing the edge set $\left\{e_{i_{1} 1}, \ldots, e_{i_{1} t}, \ldots, e_{i_{k-1} 1}, \ldots\right.$, $\left.e_{i_{k-1} t} \mid 1 \leq i_{1}<\cdots<i_{k-1} \leq n\right\}$. By analogy, there are

$$
\begin{aligned}
& \left(\begin{array}{c}
n \\
k-2
\end{array}\right)\left\{\left(\begin{array}{c}
n t-(k-2) t \\
j-(k-2) t+1
\end{array}\right)-\left(\begin{array}{c}
n-(k-2) \\
1
\end{array}\right)\left[\left(\begin{array}{c}
n t-(k-1) t \\
j-(k-1) t+1
\end{array}\right)\right.\right. \\
- & \left.\left.\left(\begin{array}{c}
n-(k-1) \\
1
\end{array}\right)\left(\begin{array}{c}
n t-k t \\
j-k t+1
\end{array}\right)\right]-\left(\begin{array}{c}
n-(k-2) \\
2
\end{array}\right)\left(\begin{array}{c}
n t-k t \\
j-k t+1
\end{array}\right)\right\} \\
= & \left(\begin{array}{c}
n \\
k-2
\end{array}\right)\left(\begin{array}{c}
n t-k t+2 t \\
j-k t+2 t+1
\end{array}\right)-\left(\begin{array}{c}
n \\
k-2
\end{array}\right)\left(\begin{array}{c}
n-k+2 \\
1
\end{array}\right)\left(\begin{array}{c}
n t-k t+t \\
j-k t+t+1
\end{array}\right) \\
+ & \left(\begin{array}{c}
n \\
k-2
\end{array}\right)\left[\left(\begin{array}{c}
n-k+2 \\
1
\end{array}\right)\left(\begin{array}{c}
n-k+1 \\
1
\end{array}\right)-\left(\begin{array}{c}
n-k+2 \\
2
\end{array}\right)\right]\left(\begin{array}{c}
n t-k t \\
j-k t+1
\end{array}\right) \\
= & \left(\begin{array}{c}
n \\
k-2
\end{array}\right)\left(\begin{array}{c}
n t-k t+2 t \\
j-k t+2 t+1
\end{array}\right)-\left(\begin{array}{c}
n \\
k-2
\end{array}\right)\left(\begin{array}{c}
n-k+2 \\
1
\end{array}\right)\left(\begin{array}{c}
n t-k t+t \\
j-k t+t+1
\end{array}\right) \\
+ & \left(\begin{array}{c}
n \\
k-2
\end{array}\right)\left(\begin{array}{c}
n-k+2 \\
2
\end{array}\right)\left(\begin{array}{c}
n t-k t \\
j-k t+1
\end{array}\right) \\
= & \sum_{i=0}^{2}(-1)^{i}\left(\begin{array}{c}
n \\
k-2
\end{array}\right)\left(\begin{array}{c}
n-k+2 \\
i
\end{array}\right)\left(\begin{array}{c}
n t-(k-2+i) t \\
j-(k-2+i) t+1
\end{array}\right)
\end{aligned}
$$


subsets of $E$ with $j+1$ elements, containing the edge set $\left\{e_{i_{1} 1}, e_{i_{1} 2}, \ldots, e_{i_{1} t}, \ldots\right.$, $\left.e_{i_{k-2} 1}, e_{i_{k-2} 2}, \ldots, e_{i_{k-2} t} \mid 1 \leq i_{1}<\cdots<i_{k-2} \leq n\right\}$ and so on. Therefore, the number of all subsets of $E$, which have $j+1$ elements and contain the edge set $\left\{e_{i_{1} 1}, \ldots, e_{i_{1} t}, \ldots, e_{i_{k-m}, 1}, \ldots, e_{i_{k-m}, t} \mid 1 \leq i_{1}<\cdots<i_{m} \leq n\right\}$, is

$$
\sum_{i=0}^{m}(-1)^{i}\left(\begin{array}{c}
n \\
k-m
\end{array}\right)\left(\begin{array}{c}
n-k+m \\
i
\end{array}\right)\left(\begin{array}{c}
n t-(k-m+i) t \\
j-(k-m+i) t+1
\end{array}\right) .
$$

Therefore, by inclusion exclusion principle, we have

$$
\begin{aligned}
f_{j} & =\left(\begin{array}{c}
n t \\
j+1
\end{array}\right)-\left(\begin{array}{l}
n \\
k
\end{array}\right)\left(\begin{array}{c}
n t-k t \\
j-k t+1
\end{array}\right) \\
& -\sum_{i=0}^{1}(-1)^{i}\left(\begin{array}{c}
n \\
k-1
\end{array}\right)\left(\begin{array}{c}
n-k+1 \\
i
\end{array}\right)\left(\begin{array}{c}
n t-(k-1+i) t \\
j-(k-1+i) t+1
\end{array}\right) \\
& -\sum_{i=0}^{2}(-1)^{i}\left(\begin{array}{c}
n \\
k-2
\end{array}\right)\left(\begin{array}{c}
n-k+2 \\
i
\end{array}\right)\left(\begin{array}{c}
n t-(k-2+i) t \\
j-(k-2+i) t+1
\end{array}\right)-\cdots \\
& -\sum_{i=0}^{m}(-1)^{i}\left(\begin{array}{c}
n \\
k-m
\end{array}\right)\left(\begin{array}{c}
n-k+m \\
i
\end{array}\right)\left(\begin{array}{c}
n t-(k-m+i) t \\
j-(k-m+i) t+1
\end{array}\right)-\cdots \\
& -\sum_{i=0}^{k-1}(-1)^{i}\left(\begin{array}{c}
n \\
1
\end{array}\right)\left(\begin{array}{c}
n-1 \\
i
\end{array}\right)\left(\begin{array}{c}
n t-(1+i) t \\
j-(1+i) t+1
\end{array}\right) \\
& =\left(\begin{array}{c}
n t \\
j+1
\end{array}\right)-\left[\begin{array}{c}
k \\
j=1
\end{array}(-1)^{k-j}\left(\begin{array}{c}
n \\
j
\end{array}\right)\left(\begin{array}{c}
n-j \\
k-j
\end{array}\right)\right]\left(\begin{array}{c}
n t-k t \\
j-k t+1
\end{array}\right) \\
& -\left[\sum_{j=1}^{k-1}(-1)^{k-1-j}\left(\begin{array}{c}
n \\
j
\end{array}\right)\left(\begin{array}{c}
n-j \\
k-1-j
\end{array}\right)\right]\left(\begin{array}{c}
n t-(k-1) t \\
j-(k-1) t+1
\end{array}\right)-\cdots \\
& -\left[\sum_{j=1}^{2}(-1)^{2-j}\left(\begin{array}{c}
n \\
j
\end{array}\right)\left(\begin{array}{c}
n-j \\
2-j
\end{array}\right)\right]\left(\begin{array}{c}
n t-2 t \\
j-2 t+1
\end{array}\right)-\left(\begin{array}{c}
n \\
1
\end{array}\right)\left(\begin{array}{c}
n t-t \\
j-t+1
\end{array}\right) \\
& =\sum_{i=0}^{k}(-1)^{i}\left(\begin{array}{c}
n \\
i
\end{array}\right)\left(\begin{array}{c}
n t-i t \\
j-i t+1
\end{array}\right),
\end{aligned}
$$

where the last equality holds by combinatorial formula $\sum_{j=0}^{k}(-1)^{k-j}\left(\begin{array}{l}n \\ j\end{array}\right)\left(\begin{array}{l}n-j \\ k-j\end{array}\right)$ $=0$.

We can now give a formula for Hilbert series of $k\left[\Delta_{s}\left(G_{l_{1}, l_{2}, \cdots, l_{n}}\right)\right]$ under the condition that the length of every cycle $G_{l_{i}}$ is $t$ for $1 \leq i \leq n$.

Theorem 4.3. Let $\Delta_{s}\left(G_{l_{1}, l_{2}, \cdots, l_{n}}\right)$ be the spanning simplicial complex of the $n$ cyclic graph $G_{l_{1}, l_{2}, \cdots, l_{n}}$, where $l_{i}=t$ for every $1 \leq i \leq n$. Then Hilbert series of 
the Stanley-Reisner ring $k\left[\Delta_{s}\left(G_{l_{1}, l_{2}, \cdots, l_{n}}\right)\right]$ is given by

$$
H\left(k\left[\Delta_{s}\left(G_{l_{1}, l_{2}, \cdots, l_{n}}\right)\right], z\right)=1+\sum_{i=0}^{d} \sum_{l=0}^{k}(-1)^{l}\left(\begin{array}{c}
n \\
l
\end{array}\right)\left(\begin{array}{c}
n t-l t \\
j-l t+1
\end{array}\right) .
$$

Proof. From [4, Corollary 5.4.5], we know that if $\Delta$ is a simplicial complex and $f(\Delta)=\left(f_{0}, \ldots, f_{d}\right)$ is its $f$-vector, then the Hilbert series of the Stanley-Reisner ring $k[\Delta]$ is given by

$$
H(k[\Delta], z)=\sum_{i=-1}^{d} \frac{f_{i} z^{i+1}}{(1-z)^{i+1}}, \quad d=\operatorname{dim} \Delta .
$$

The desired formula follows from the theorem above at once.

Acknowledgment. The authors are grateful to Professor Zhongming Tang for useful discussions. They would like to express their sincere thanks to the editor for help and encouragement. Special thanks are due to the referee for a careful reading and pertinent comments.

\section{References}

[1] I. Anwar, Z. Raza and A. Kashif, Spanning simplicial complexes of uni-cyclic graphs, arXiv:1311.0981v1.

[2] W. Bruns and J. Herzog, Cohen-Macaulay Rings, Revised Edition, Cambridge Studies in Advanced Mathematics, 39. Cambridge University Press, Cambridge, 1998.

[3] F. Harary, Graph Theory, Reading, MA: Addison-Wesley, 1994.

[4] R. H. Villarreal, Monomial Algebras, Monographs and Textbooks in Pure and Applied Mathematics, 238. Marcel Dekker, Inc., New York, 2001.

[5] G. Zhu, F. Shi and Y. Geng, Spanning simplicial complexes of n-cyclic graphs with a common edge, Int. Electron. J. Algebra, 15 (2014), 132-144.

Yan Pan, Rong Li and Guangjun Zhu

School of Mathematical Sciences

Soochow University

Suzhou 215006, China

e-mails: panyan19921120@126.com (Y. Pan)

lrbjxda@126.com (R. Li)

zhuguangjun@suda.edu.cn (G. Zhu) 